\title{
Portable IoT NIR Spectrometer for Detecting Undesirable Substances in Forages of Dairy Farms
}

\author{
Guillermo Rego Fernández, Jorge Losada Matías, Francisco \\ Ferrero, Marta Valledor, Juan Carlos Campo \\ Dept. of Electrical, Electronic, Computers, and System \\ Engineering \\ Universidad de Oviedo, Gijón, Spain \\ ferrero@uniovi.es
}

\author{
Luis Royo, Ana Soldado, Sergio Forcada \\ Dept. of Nutrition, Grasslands and Forages \\ Regional Institute for Research and Agro-Food Development \\ Villaciciosa, Spain \\ asoldado@serida.org
}

\begin{abstract}
Food security and the agriculture sustainable development are two important challenges that arise in the Common Agricultural Policy (CAP) of the European Union. However, oftentimes, the necessary equipment to fully these requirements is expensive and complex to use. In this paper, a relatively low-cost prototype instrument is presented for the detection of contaminants and pathogens in forages, using near infrared spectrometry (NIRS) techniques. Through the Internet of Things (IoT) tools, data are sent to the cloud for processing while being accessible for any device. All this opens up new possibilities, and new challenges, for the sustainable management of natural resources.
\end{abstract}

Keywords-internet of things, spectrometry, near infrared, ESP32, bluetooth low energy, chemometric model.

\section{INTRODUCTION}

Standards for food safety are increasingly strict, which is something that any farm, however small, must consider to be competitive in the current market. As such, European Directive 2002/32/CE regulates undesirable substances and their maximum levels to protect human health, animal health, and the environment. The cattle management system shows that there are very few, and sometimes nonexistent, actions related to control parameters of chemical and sanitary contamination of forages and organic resources (slurry, manure), produced on the farm itself. Furthermore, it is only when the effects on animals or humans are observed that the focus of contamination (mycotoxins, dioxins, E. coli, etc.) is searched for.

This fact is related to the difficulty in measuring the degree of contamination, based on the high price of the analysis and the lengthy time of sample preparation. In this sense, it would be advisable to have analytical procedures in place that would enable the reduction of costs and the taking of simple, quick, and, if possible, "in situ" measures.

The current challenge is the development of instruments that, based on the selection and identification of specific spectral bands according to certain pathogens and/or relevant contaminants, such as heavy metals, polycyclic aromatic hydrocarbons (PAHs), and pathogens, can determine the quality of animal waste, soils, animal feed, and milk production. Fig. 1 shows the main polluting substances present in the agro-livestock production chain and the proposed control strategies.

The financial support of the Spanish Government (Ministry of Economy, Industry and Competitiveness), Project RTA2015-00061-C0202 , is gratefully acknowledged.

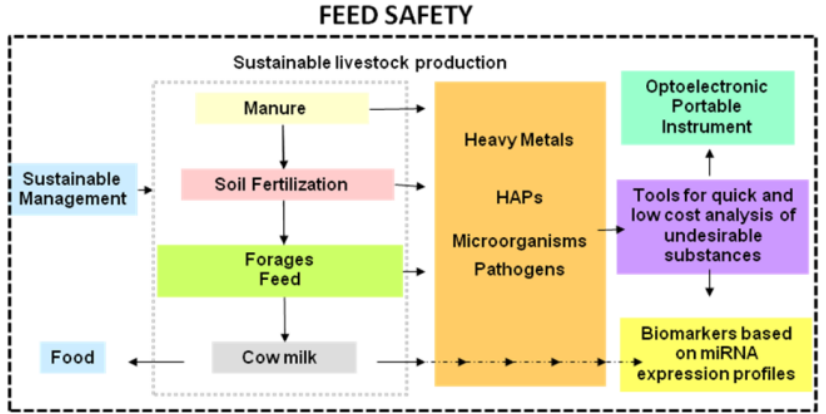

Fig. 1. Undesirable substances in the agro-livestock production chain and proposed control strategies.

Specially, the presence of pathogenic microorganisms in the forages of animals is a high risk, both for livestock production and for health, whether human or animal. Therefore, a series of controls must be carried out to minimize this risk. Avoiding contamination sources is always less expensive than carrying out pollution elimination tasks.

Nowadays, there are instruments that can do the task of detecting these unwanted microorganisms. However, their price is very high, they require specialized personnel, and they are not usually portable. That said the nature of livestock farms makes it necessary for many controls to be done "in situ." Therefore, there is a need to develop a portable instrumentation of relatively low cost and with a simple operation for the owner of the farm. The evolution of technology in the field of near infrared (NIR) spectrometry allows to use measurement methods that are much cheaper and faster than those already in existence.

Besides the price and the portability, there is the problem of the distribution of the data. Existing equipment cannot extract the data simply because it does not usually have the appropriate connectivity. This causes a problem when following the evolution of the data. This information can be very useful for the owner or administration responsible for food safety. Having a data history is always an added benefit that can give valuable information about what is happening on the farm. In this sense, the advance in communication technologies and smartphones opens up a range of new possibilities that could be applied to develop a solution befitting the desired conditions. The proposed solution addresses this problem through the new technologies that have appeared thanks to the IoT.

The Bluetooth Low Energy (BLE) offers low consumption, which is necessary in applications powered by batteries, has $30 \mathrm{~m}$ of theoretical scope, and can reach speeds of $1 \mathrm{Mbit} / \mathrm{s}$. The smartphone will give the 
possibility of creating a graphical interface to interact with the user, in addition to providing the Internet access to reach the cloud. In it, the data can be treated to be accessible and visualizable from any device with access to them.

The remainder of the article is structured as follows. Related work is covered in Section II. Section III presents the materials. Section IV shows the implementation of the IoT measurement system. The data processing is addressed in Section V. Finally, Section VI offers a conclusion.

\section{RELATED WORK}

Numerous publications and much scientific research support the efficacy of the analysis in the visible (VIS)near infra-red (NIR) wavelength range for the quantification of quality and safety parameters in animal feed [1-7]. All this work has been carried out within the framework of near-infrared spectroscopy (NIRS) as a rapid diagnostic tool, allowing to evolve from laboratory equipment to portable commercial equipment for determining the quality [8] and sanitary hygienic conditions of food [9]. This commercial equipment is expensive, and it only works with specific commercial software. The lower-cost equipment has optical and electronic characteristics that do not allow the collection of quality spectral information from the matrices of this study. For these reasons, the development of a portable, relative low-cost NIR prototype, with instrumental characteristics optimized for solving the problem at hand, is proposed.

\section{MATERIALS}

Spectroscopy is a measurement technique that enables the recognition and characterization of physical materials through the variation in the absorption or emission of different wavelengths of light. Spectroscopy can be performed with visible, infrared, or ultraviolet wavelengths. The NIR and short wave infrared (SWIR) regions are particularly rich in information on molecular vibration modes; as such, this range of wavelengths is used in molecular analysis applications.

All spectrometer designs have five basic components: radiation source, spectral wavelength selection system, sampling device, detector, and signal processor [10-14]. Different configurations of commercial NIR portable equipment are available, which have been evaluated as showing serious limitations related to window size, the high cost of the instrument, and errors in cross-validation between $2 \%$ and $6 \%$ in absolute value.

The most common sources of light are still tungsten lamps, while in the case of detectors, portable devices are usually used from charge-coupled devices with diffraction gratings to arrays of photodiodes with linear filters [15-17]. All these configurations necessarily involve small window sizes or high costs, which are only acceptable in laboratory equipment; this is an important restriction for applications in the field.

A novel spectroscopic solution is that proposed by Texas Instruments, based on the digital light processing (DLP) technology. Fig. 2 shows this solution [18]. The differentiating element is the digital micromirror device
(DMD), which consists of an array of hundreds of thousands of micromirrors. By activating or not activating the mirror columns, only the desired wavelengths are reflected to the detector. In the IR/NIR wavelength region, this allows the use of a high-performance, cost-effective single element detector, while providing wavelength selection agility, speed, and mechanical stability [19].

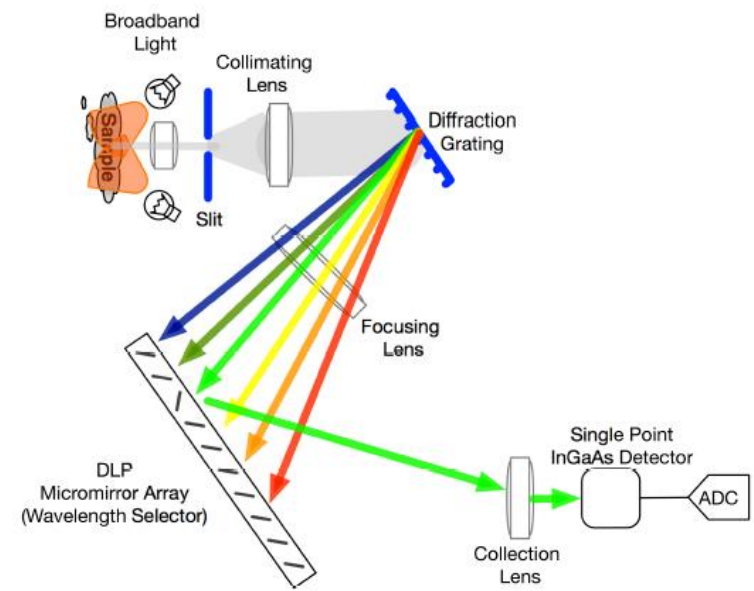

Fig. 2. Spectrometer using a DLP DMD and a single element detector.

\section{A. DLP NIRscan Nano}

Texas Instruments provides a complete evaluation module to design a high performance, affordable NIRS. In this work, the NIRscan Nano DLP evaluation module is used. It is a compact battery-operated evaluation module for portable NIRS solutions. It supports BLE to enable mobile lab measurements for hand-held spectrometers. The EVM incorporates the DLP2010NIR DMD, a diffraction grating, and a single element detector to replace expensive InGaAs linear array-based detector designs. The sensing range is between $900 \mathrm{~nm}$ and $1700 \mathrm{~nm}$. The TI embedded processor commands the DMD controller to turn on only the precise mirrors which are illuminated by the specific wavelengths of light to be measured at each instant of time. The easy programmability of the processor allows users to command specific column widths (wavelength ranges) or other patterns for their spectroscopy requirements. Fig. 3 shows an image of the DLP NIRscan Nano.

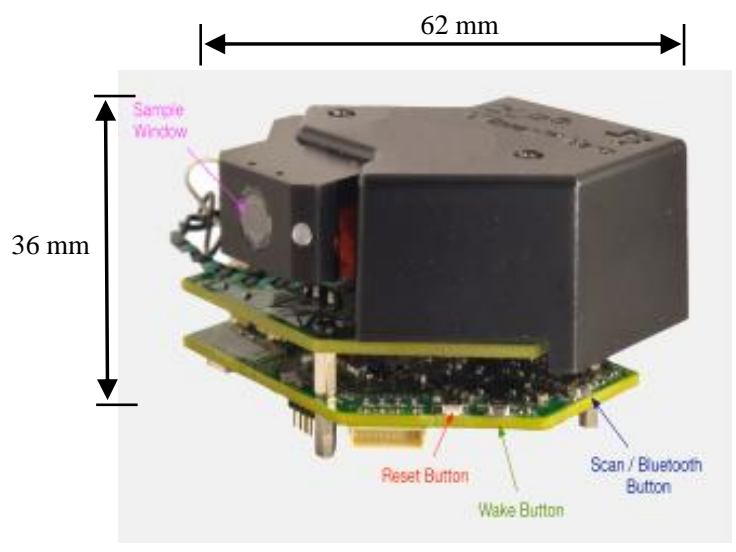

Fig. 3. DLP NIRscan nano rear view.

\section{B. Development board for IoT}

In order to send the data to the cloud a WiFi \& Bluetooth board (LOLIN D32) based on the ESP-32 
microcontroller chip was used. It is especially suitable for IoT applications due to its very low consumption. For the connection this board to the NIRscan Nano DLP module, a printed circuit board was developed and attached to the spectrometer, as shown in Fig. 4. On this board, the pins are connected to the 5VDC servomotor that moves the sample holder. A DC-DC converter (Pololu U1V10F5) is used to convert the $3.3 \mathrm{~V}$ from the battery to $5 \mathrm{~V}$ to power the servomotor.

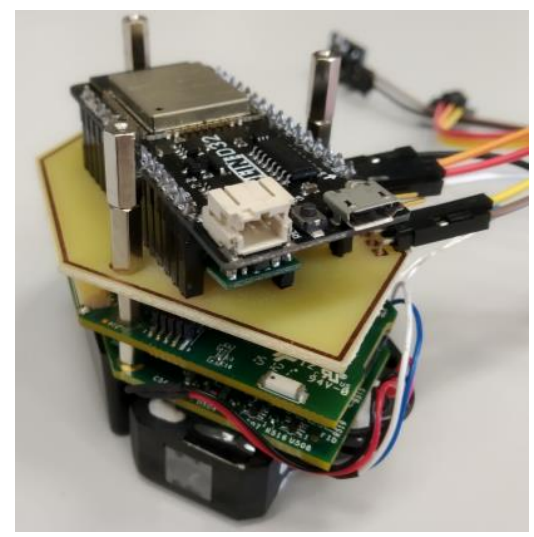

Fig. 4. Printed circuit board for connecting the LOLIN D32 board to the NIRscan Nano DLP module.

\section{IOT MESUREMENT SYSTEM}

Figure 5 shows the general scheme of the proposed measurement system. It consists of the DLP NIRscan Nano module and a board based on the well-known ESP32 module, the microcontroller of which will be responsible for turning the servomotor where the sample holder will be placed. The spectrometer will make several measurements when the sample holder is rotating, which will make the measurements as homogeneous as possible. These data can be sent to a mobile application via Bluetooth, which is integrated into the NIRscan. There is also the option of sending them to the computer via a USB connection. The final objective is to send these data to the cloud, where the spectra of the analyzed substances can be treated with more powerful tools, confirming or rejecting the existence of pathogenic microorganisms in the sample. In addition, the results may be accessible from other mobile devices with Internet access.

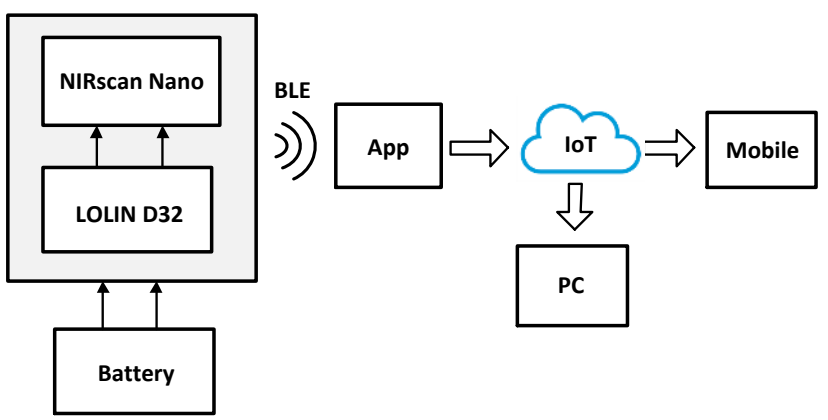

Fig. 5. General scheme of the proposed IoT NIR spectrometer.

The small size of the sample window $(10 \mathrm{~mm} \times 10 \mathrm{~mm})$ makes difficult to directly measure the samples. To avoid this drawback, a semi-circular sample holder was attached on the spectrometer, as shown in Fig. 5. This holder rotates and, in each position, six measurements are made. For the rotation of the sample holder, a servomotor controlled from the ESP32 was used. In addition to the rotation control, the ESP32 has two buttons, one to start the measurements and another to set the load position of the sample holder. When the start button is pressed, the servomotor will begin to turn, stopping at 10 different positions where the measurements will be made. Once the sample holder reaches the $180^{\circ}$ position, it returns to the initial position, where it waits to make a new measurement. When the load button is pressed, the servomotor will go to an intermediate position of about $90^{\circ}$, facilitating the loading and unloading of the sample. When the device is not performing either of these two actions, the microcontroller will go into sleep mode to minimize power consumption.

Autonomy is fundamental in any portable IoT device. A $3.7 \mathrm{~V}, 1800 \mathrm{mAh}$ Lithium Polymer battery is used to power the entire system, providing an estimated autonomy of 15 hours when it is at rest. A $10 \mathrm{k} \Omega$ NTC thermistor is required to safely charge the battery and monitor its temperature. When the measurement is being made, the spectrometer alone consumes $500 \mathrm{~mA}$, but the measurement time is only about $16 \mathrm{~s}$. So, if the user made an average of 10 measurements per hour, the autonomy of the prototype will be 12 hours (if it is on all the time). Texas Instruments provides free software and firmware downloads to give developers flexibility and advanced control of the DLP NIRscan Nano. Many of the parameters of the spectrometer can be modified, increasing the flexibility of the measurements, as can be seen in Fig. 7 .
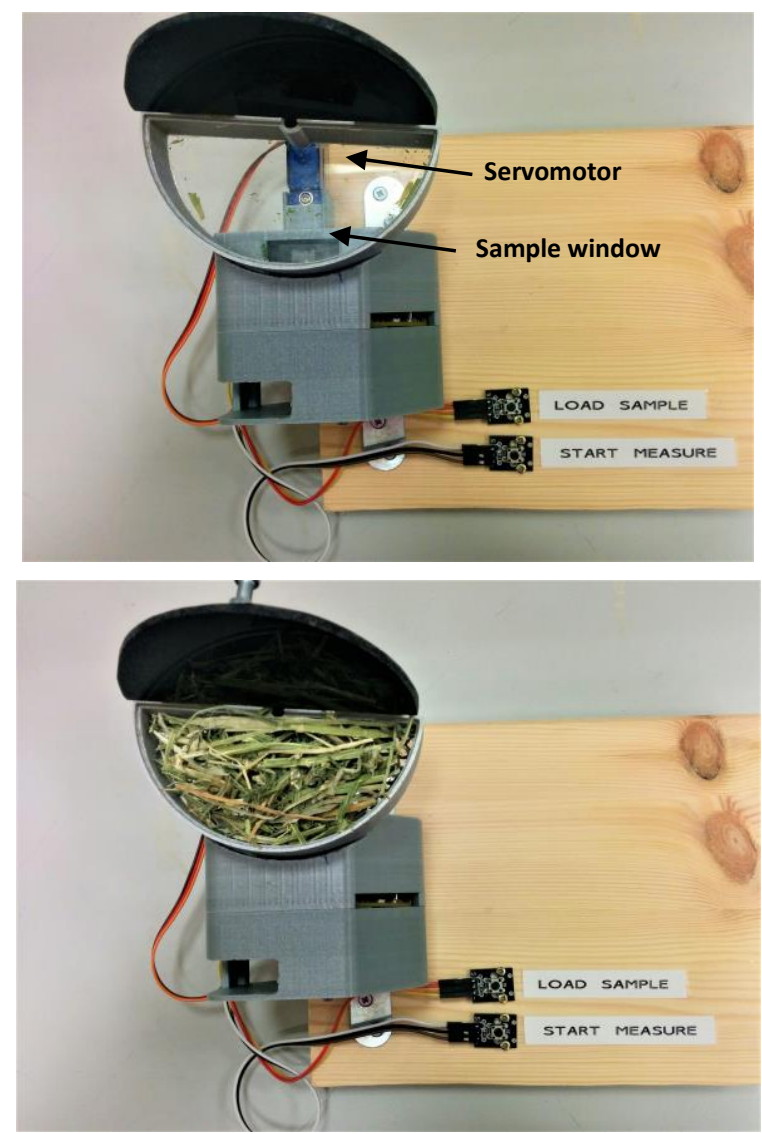

Fig. 6. NIRS prototype for the measurement of contaminants in forages. 


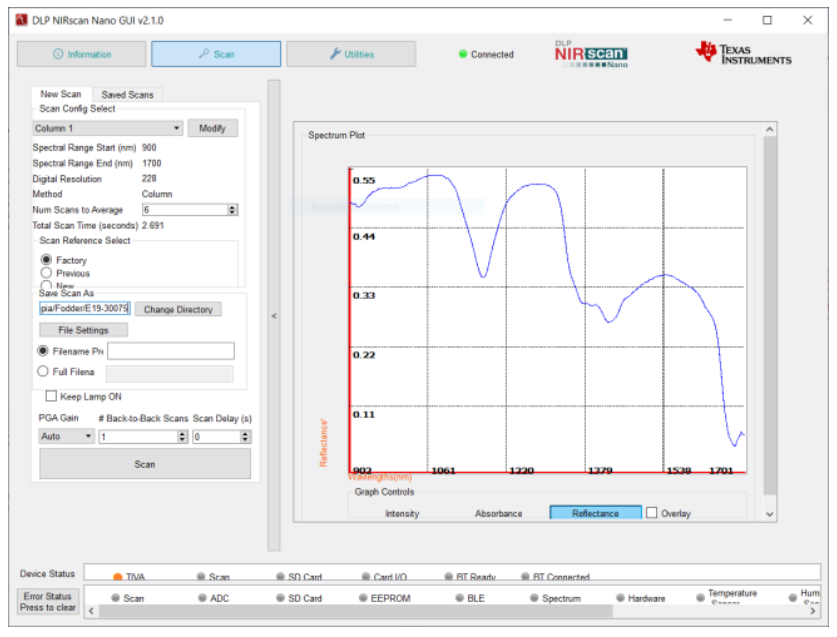

Fig. 7. Texas Instruments software for the NIRscan.

\section{DATA PROCESSING}

After performing the measurement, the spectrometer will send the data via BLE to the mobile device with which it is paired. Once there, the data of the spectra will be sent to the cloud, where the statistical treatment will be realized. Using the Python language, a dashboard has been devoloped to display the data and allow the user to upload and download the spectra. The data must be in "csv" format, which is the one provided by the spectrometer. The dashboard will be in charge of making the average of the 10 spectra and showing on a graph the median of the sample. Fig. 8 shows the spectra of a sample of alfalfa in 10 points and the median of spectra. In order to obtain the repeatability of the instrument 10 consecutive spectra were obtained, as shown in Fig. 9. The repeatability is $3.9 \%$, which was calculated as the ratio of the maximum difference among spectra to the maximum value of reflectance.

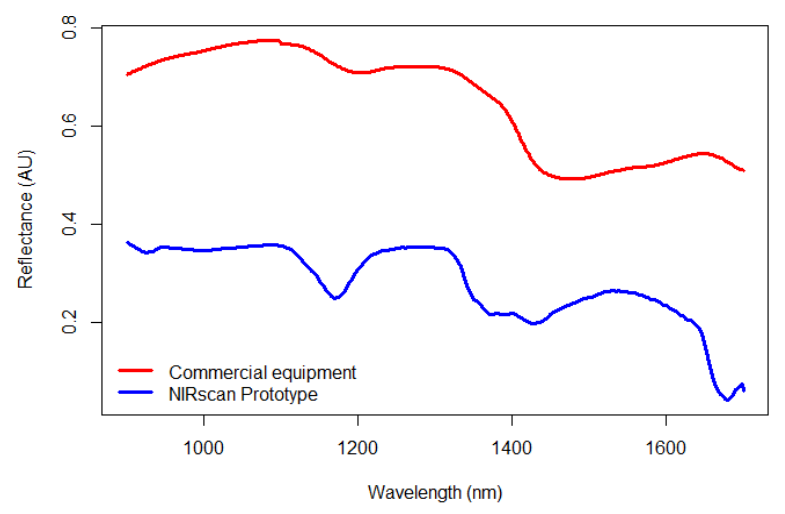

Fig. 8. Spectra of a sample of alfalfa in 10 points and the median.

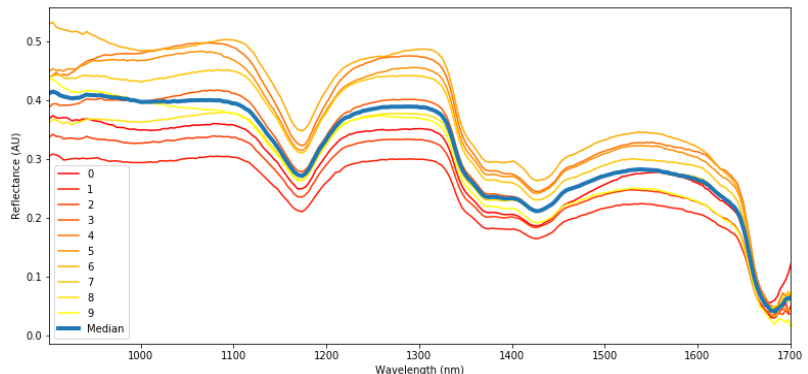

Fig. 9. Spectra of a sample of alfalfa to calculate the repeatability of the instrument.
The next task is to develop and implement a chemometric model in the instrument for the detection and quantification of undesirable substances and pathogens in the matrices. Fig. 10 shows the process to obtain the chemometric model. Firstly, the data is pre-processing applying a Normal Standard Variate (SNV), to eliminate the problems of scattering. Secondly, the Savitzky-Golay digital filter is applied to preserve characteristics of the initial distribution such as the relative maximums and minimums, as well as the width of the peaks.

The model will be obtained from a regression used in NIR measurements, called Partial Least Squares Regression (PLS). With this model, it will be possible to detect if a sample is contaminated or its nutritional properties. Currently, the model is not finished, however there are already some nutritional values that can be predicted quite well, such us the Neutral Detergent Fiber (NDF). It is the most common measure of fiber used for animal feed analysis. Fig. 11 shows the standard error of cross validation (SECV) and the determination coefficient of cross validation (1-VR) obtained for NDF.

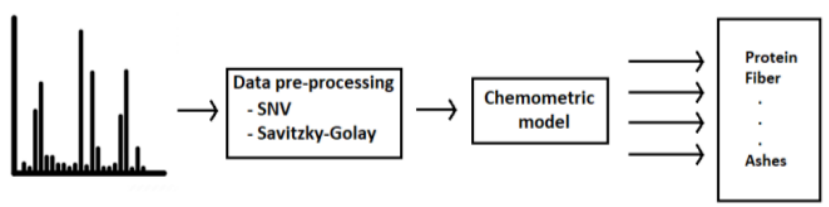

Fig. 10. Process to obtain the chemometric model.

\begin{tabular}{|c|c|c|c|c|c|c|}
\hline \multirow{2}{*}{\multicolumn{2}{|c|}{ Constituent }} & \multicolumn{2}{|l|}{ FND } & \multirow[b]{2}{*}{ Range } & \multirow{3}{*}{$\begin{array}{l}29.24-61.01 \\
1-\mathrm{vR}\end{array}$} & \multirow{3}{*}{ Standard deviation 5.309} \\
\hline & 41.472 & & & & & \\
\hline & SEC & RSo & $\mathrm{F}$ & SECV & & \\
\hline 1 & 3.676 & 0.521 & 48.78 & 3.932 & 0.466 & \\
\hline 2 & 3.179 & 0.641 & 15.50 & 3.560 & 0.562 & \\
\hline 3 & 2.847 & 0.712 & 11.34 & 3.327 & 0.617 & \\
\hline 4 & 2.473 & 0.783 & 14.35 & 2.962 & 0.697 & \\
\hline
\end{tabular}

Fig. 11 Model characteristics for neutral detergent fiber.

The provider used to host the application will be Amazon Web Services (AWS). Fig. 12 shows the dashboard structure on the cloud. To facilitate its deployment in other platforms the developed application will be running inside a Docker container. The user, using the web browser, where the dashboard will be displayed, will communicate with the cloud through the free and open source software, Nginx. This will work as a web server and will allow him to communicate with the application itself, giving the system a layer so that the data is not directly exposed. After that, a Python WSGI HTTP Server for UNIX will be found (Gunicorn). The application will be running under this software. It has been developed using Dash, which is a framework for building analytical web applications. The data spectra will be stored in a database, a non-relational and open source database, called MongoDB.

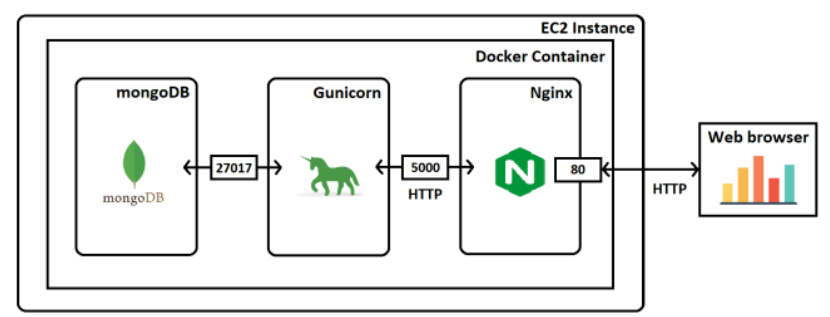

Fig. 12 Dashboard structure on the cloud. 


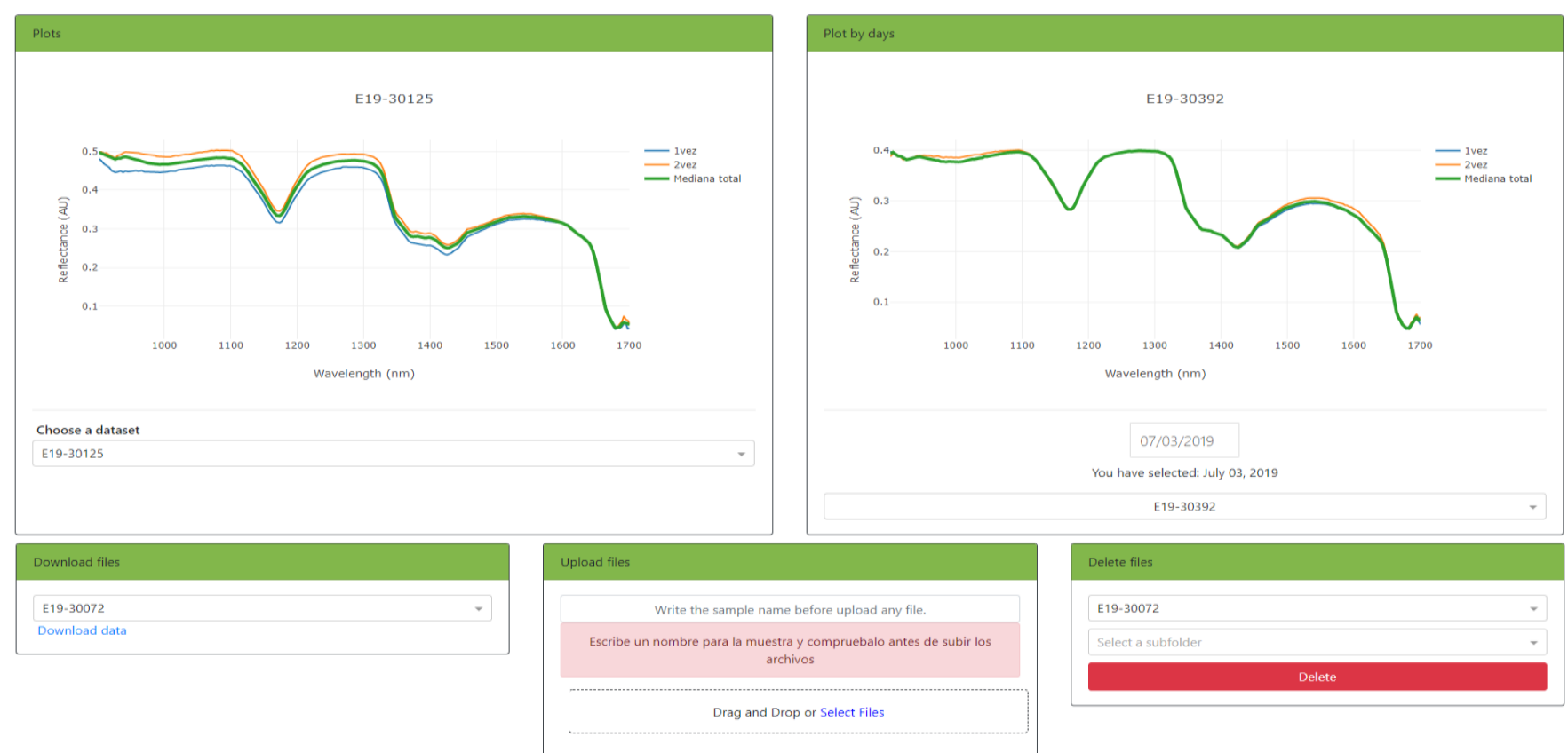

Fig. 13. Dashboard to interact with the user.

Finally, the treated data will be displayed in a dashboard where the user can see the results and the graphics generated, as can be seen in Fig. 13.

Deploying the entire system in the cloud will allow adapting to new volumes of data in the future with relative ease, in such a way that any authorized person can access from anywhere in the world if they have access to the Internet and not depend on the construction and maintenance of own servers, which makes a very low initial cost.

\section{CONCLUSION}

This work presents a prototype of a portable IoT NIR spectrometer for detecting undesirable substances in forages of dairy farms. It is based on the Texas Instruments NIRscan Nano DLP evaluation module and the ESP-32 microcontroller chip, which are especially suitable for IoT applications. This instrument can be used at farm level as a safety and quality tool to prevent contamination risks for animals and humans, and the losses that it entails. It could also allow the competent authority to monitor each farm avoiding risks for public health. The next step will be to use chemometric strategies to improve the understanding of chemical information and to correlate quality parameters or physical properties to analytical instrument data.

\section{REFERENCES}

[1] Williams P. and Norris K. Eds. Book: Near infrared technology in the agricultural and food industry, second edition. ISBN 978-1891127-24-3. 2001.

[2] Decruyenaere V., Lecomte Ph., Demarquilly C., Aufrere J., Dardenne P., Stilmant D., Buldgen A. "Evaluation of green forage intake and digestibility in ruminants using near infrared reflectance spectrscopy (NIRS): Developing a global calibration". 200). Anim Feed Sci. Technol. , Volume 148, Issues 2-4, 138-156.

[3] Baeten, V., Manley, M., Fernandez Pierna, J.A., Downey, G. \& Dardenne, P. Spectrometric Technique: Fourier Transform Mearinfrared (FT-NIR) Spectroscopy. In: Modern Techniques for Food
Authentication, Sun, Da-Wen. Dublin - UK, Elsevier, 2008. 117147.

[4] De la Haba M.J., Fernández Pierna J.A., Fumière O., A. GarridoVaro, J.E. Guerrero, D.C. Pérez-Marín, P. Dardenne and V. Baeten. "Discrimination of fish bones from other animal bones in the sedimented fraction of compound feeds ny near infrared microscopy (NIRM)". J. of NIRS, 15. 2007, 81-88.

[5] Fernández Pierna J.A., V. Baeten, P. Dardenne. "Screening of compound feeds using NIR hyperspectral data". Chemometrics and Intelligent Laboratory Systems, 84. 2006, 114-118.

[6] Fernández-Ibáñez V., Soldado A., Vicente F., Martínez-Fernández A., De La Roza-Delgado B.Particle size optimisation in development of near infrared microscopy methodology to build spectral libraries of animal feeds. 2008. Journal of Near Infrared Spectroscopy 16, 243-248M. Young, The Technical Writer's Handbook. Mill Valley, CA: University Science, 1989.

[7] Okparanma R.N., Mouazen A.M. "Visible and Near-Infrared Spectroscopy Analysis of a Polycyclic Aromatic Hidrocarbon in Soils". The Scientific World Journal. vol. 2013.

[8] Teixeira Dos Santos C.A., Lopo M., Páscoa R.N.M.J., Lopes J.A., "A review on the applications of portable near infrared spectrometers in the agrofood ndustry". Appl. Spec. 67(11), 2013, 1215.

[9] Prado N., Fernández-Ibáñez V., González P., Soldado A., "On-site NIR spectrsocopy to control shelf life of pork meat". Food Anal Methods 4(852), 2011, 589.255-291.

[10] Blanco M., Villaroya I., "NIR spectroscopy: a rapid-response analytical tool", Trends in Analytical chemistry. 2002. vol. 21, nº pp. 240.250 .

[11] Roggo Y., Chalus P., Maurer L., Lema-M. C. "A review of near infrared spectroscopy and chemometrics in pharmaceutical technologies". J. of Pharamaceutical and Biomedial Analysis. 2007. 44, pp. 683-700.

[12] Siesler H.W., Ozaki Y., Kawata S., Heise H.M., "Near-Infrared spectroscopy", Ed. Wiley-VCH, 2002.

[13] Osborne B. G., Fearn T., Hindle P.H., "Practical NIR spectroscopy with applications in food and beverage analysis", Prentice-Hall, 1993.

[14] González-Martín I., Álvarez-García N., Hernández-Andaluz J.L., "Instantaneous determination of crude proteins, fat and fibre in animal feeds using near infrared reflectance spectroscopy technology and a remote reflectance fibre-optic probe", Animal Feed Science and Technology. 2006 vol. 128, Is 1-2, pp. 165-171.

[15] Larrain M., Guesalaga A.R., Agosin E., “A Multipurpose Portable Instrument for Determining Ripeness in Wine Grapes Using NIR 
Spectroscopy", IEEE Trans. on Instrumentation and Measurement. 2008. Vol: 57, N 2, pp. $294-302$.

[16] Temma T., Hanamatsu K., Shinoki F., "Development of a portable near infrared sugar-measuring instrument," J. Near Infrared Spectrosc. 2002. 10(1), pp. 77-83.

[17] Landau S., Glasser T., Dvash L., "Monitoring nutrition in small ruminants with the aid of near infrared reflectance spectroscopy (NIRS) technology: A review Review Article", Small Ruminant Research. 2006. 61, Issue 1, Pages 1-11.

[18] DLP NIRscan ${ }^{\mathrm{TM}}$ Nano EVM User's Guide. User's Guide. Texas Instruments. 2017.

[19] DLP Technology for Spectroscopy. White Paper DLPA048AFebruary 2014-Revised August 2016. 